\title{
Obesity - The Epidemic like no other in human history
}

Obesity is defined as abnormal or excessive fat accumulation which is associated with adverse health outcomes. ${ }^{1}$ Over the past few decades, Obesity has become a major public health issue with epidemic proportions that affects people across all ages, both genders.Globally in 2015, approximately 604 million adultsand 108 million children were obese. The prevalence of obesity is increased in almost all countries since 1980 and a doubling in prevalence in 70 countries. ${ }^{2}$ It is a frightening fact that the rate of increase in obesity in childhood in many countries has been greater than the rate of increase in adults.

The percentages of Sri Lankan adults in the overweight, obese and centrally obese categories were $25.2 \%, 9.2 \%$ and $26.2 \%$, respectively.In addition, female sex, urban living, higher education, higher income and being in the middle age were shown to be associated with overweight and obesity in Sri Lankans. $^{3}$

In majority of cases, Obesity is due to a positive energy balance rather than a secondary cause. Excess calorie intake from any source (eg- refined carbohydrates, processed meat, fast foods) and sedentary life style causing reduced energy expenditure leads to weight gain and obesity. Prolonged time spent in front of screens such as television, laptop and smartphones is a major risk factor associated with obesity. It is a false belief that genetic factors play a significant role in the development of obesity. High prevalence of obesity among first degree relatives is observed because they share a common dietary pattern and life style rather than the identical genes.

Obesity is associated with conditions such as diabetes mellitus, hypertension, dyslipidaemia, Ischemic heart disease, stroke, obstructive sleep apnoeaand osteoarthritis. In young females, obesity leads to polycystic ovarian syndrome in which subfertility is a major feature. Furthermore, obese subjects are often stigmatized in education, employment and healthcare. Obesity has a negative impact on the productivity of an individual as well. In a meta-analysis including over 30 million people, obesity was associated with increased all-cause mortality. ${ }^{4}$

Body Mass Index (BMI) is the most commonly used anthropometrical measurement in the assessment of obesity. In Asian population, Overweight and Obesity are considered if BMI 23-24.9 and $\geq 25 \mathrm{~kg} / \mathrm{m} 2$ respectively. However, BMI doesn't provide any information regarding central adiposity which is anindependent risk factor for cardiovascular disease andoverall mortality. ${ }^{5} \mathrm{~A}$ waist circumference $\geq 80 \mathrm{~cm}$ ( 31 inches) in Asian females and $\geq 90 \mathrm{~cm}$ (35 inches) in Asian males is considered abnormaland provides independent risk information on obesity that is not accounted for by BMI. 
Adult criteria used in the assessment of obesity can not be applied in children because they grow in height as well as weight. Therefore sex specific BMI for age charts available in the Sri Lanka Child Health Development Record (CHDR) should be used to asses obesity in children. Childhood overweight and obesity categories are defined if BMI between +1SD to +2SD and more than +2SD for age and sex respectively.

The important components of a weight loss programme are dietary modification, exercise and behavior therapy. Special attention should be paid to behavior therapy which helps patients to make long term changes in their eating behavior and control stimuli that trigger overeating. Setting up realistic goals and frequent reinforcement are the principal determinants of a successful weight loss programme. Web based appsand weight loss programmes/schedules can be used effectively in obese individuals who are competent in information technology.

Drug therapy in the management of obesity is only complimentary for diet, exercise and behavior therapy and initiation of such intervention should be decided on individual basis. Currently FDA approved medications for weight loss includesorlistat, lorcaserin, liraglutide, naltrexone-buproprion and phentermine-topiramatecombinations. ${ }^{6}$ Due to lack of evidence, liposuction, dietary/herbal supplements and acupuncture are not recommended for weight reduction.

Bariatric surgery is considered as the most effective method in the management of obesity. Compared to medical therapy, bariatric surgery is superior in achieving remission in diabetes and improving cardiovascular outcome and survival in obese subjects. ${ }^{7}$ Candidates for bariatric surgery include adults aged 18-65 years who have a $\mathrm{BMI}>35 \mathrm{~kg} / \mathrm{m}^{2}$ without any comorbid factors or a BMI $>30 \mathrm{~kg} / \mathrm{m}^{2}$ with at least one comorbid factor. ${ }^{8}$ Types of bariatric surgical procedures include sleeve gastrectomy, mini gastric bypass, gastric banding and Roux-en-Y gastric bypass.

We, as a society should make a collaborative effort to tackle current epidemic of obesity. First step is to change the attitudes towards obesity. Some in our society still consider obesity as a symbol of prosperity and wealth. We should move away from this attitude and consider obesity as a sign of illhealth. During pregnancy and lactation, women gain significant weight due to the belief that they need to significantly increase their food intake to accommodate both the mother andfetus.It is the healthcare provider's responsibility to advice mothers on adequate balanced nutrition and target weight gain in individual basis rather than blanket advice during this period.

Calorie conscious society is an important concept in reducing the burden of obesity. From childhood onwards, all individuals should be educated on their calorie requirement and intake with a special attention in providing information about calorie content of various foods \& meals and methodology of calorie intake calculation. Currently, calorie contents of traditional Sri Lankan foods and meals are not freely available and necessary steps need to be taken to provide such information to general public. Development of web based apps/programmestailor-made for Sri Lankan society, which calculate calorie intake and expenditure is an attractive way to create calorie awareness among young generation. 
It is necessary to develop infrastructure facilities which benefit people to engage in exercise and sports throughout the country. Cycling should be encouraged as a mode of transport and it has added benefits such as reduction in fuel consumption and air pollution.

As leaders in healthcare delivery, it is our duty to create awareness about the epidemic of obesity and grab every opportunity in individual patient consultations, workplaces and social gatherings to educate general public on how to overcome obesity.

As smoking, obesity does kill people. Let's confront obesity withserious intent and full force.

References

1. World Health Organization: Obesity: Preventing and managing the global epidemic; WHO technical report series 894. World Health Organization, Geneva; 2000.

2. GBD 2015 Obesity Collaborators, Afshin A, Forouzanfar MH, et al. Health Effects of Overweight and Obesity in 195 Countries over 25 Years. N Engl J Med 2017; 377:13.

3. Katulanda $\mathrm{P}$, Jayawardena MA, Sheriff $\mathrm{MH}$, et al. Prevalence of overweight and obesity in Sri Lankan adults. Obes Rev 2010 ;11(11):751-6.

4. Aune D, Sen A, Prasad M, et al. BMl and all cause mortality: systematic review and nonlinear dose-response meta-analysis of 230 cohort studies with 3.74 million deaths among 30.3 million participants. BMJ 2016; 353:i2156.

5. Jensen MD, Ryan DH, Apovian CM, et al. 2013 AHA/ACC/TOS guideline for the management of overweight and obesity in adults: a report of the American College of Cardiology/American Heart Association Task Force on Practice Guidelines and The Obesity Society. Circulation 2014; 129:S102.

6. Daneschvar, Homayoun L, Aronson MD, et al. FDA-Approved Anti-Obesity Drugs in the United States The American Journal of Medicine, Volume 129, Issue 8, 879.e1 - 879.e6

7. Schauer PR, Bhatt DL, KirwanJP, et al. Bariatric surgery versus intensive medical therapy for diabetes 3 year outcomes. N Engl J Med 2014;370: 2002-13.

8. Mechanick JI, Youdim A, Jones DB, et al. Clinical practice guidelines for the perioperative nutritional, metabolic, and nonsurgical support of the bariatric surgery patient - 2013 update: cosponsored by American Association of Clinical Endocrinologists, the Obesity Society, and American Society for Metabolic and Bariatric Surgery. EndocrPract 2013; 19(2): 337-72. 